\title{
Solar Cell Power Conversion Efficiency Dependence on Multilayered Si and ZnO QD films
}

\author{
A Cordova-Rubio ${ }^{1,2}$, R Lopez-Delgado ${ }^{1,2}$, A Zazueta-Raynaud ${ }^{1,2}$, Velásquez $\mathrm{O}^{3}, \mathrm{M} \mathrm{E} \mathrm{Alvarez-Ramos}^{2}$ \\ and A Ayon ${ }^{1}$ \\ 1. U. of Texas at San Antonio, Dept. of Physics and Astronomy, MEMS Research Lab, One UTSA \\ Circle, San Antonio, TX. \\ 2. Universidad de Sonora, Depto. de Física, Blvd. Luis Encinas y Rosales, Col. Centro, Hermosillo, \\ Sonora, México. \\ 3. Centro de Investigación en Nanociencia y Nanotecnología Universidad de Guadalajara, Ameca, \\ Jalisco México.
}

In recent years, solar cells have typically been made of silicon because the efficiency that these photovoltaic devices present is relatively high [1,2]. However, it has some limitations in the range of energies of photon absorption because only photons with energy greater than the bandgap can be absorbed by the material. The aforementioned shortcoming could be addressed by using photoluminescent quantum dots that exhibit a Stokes shift $[3,4]$ as a method to improve the spectral response of short wavelength photons in silicon solar cells by absorbing photons in the range of 300-450 $\mathrm{nm}$ and re-emitting in the range of 500-600 $\mathrm{nm}$ where the device exhibits a better response. To this end. we report the synthesis and characterization of silicon $(\mathrm{Si})$ and zinc oxide $(\mathrm{ZnO})$ quantum dots (QDs), that exhibit the aforementioned down-shifting photoluminescent characteristics, and their deployment on photovoltaic devices. The Si QDs were synthesized in a water-based colloidal solution [4] and for their application in the photovoltaic devices, they were deposited directly on the surface of solar cells.

Additionally, ZnO QDs were synthesized in an ethanol-based colloidal solution employing a controlled precipitation method [5] and were subsequently dispersed in a matrix of polymethyl methacrylate (PMMA) prior to being deployed on solar cells for the corresponding absorption and photoluminescent response characterization. Si QDs exhibited a photoluminescence peak centered around $540 \mathrm{~nm}$ as can be seen in Figure 1(a), while ZnO QDs had a photoluminescent peak centered around $530 \mathrm{~nm}$ as shown in Figure 1(b). The thickness of the photoluminescent coatings for silicon solar cells was theoretically optimized to minimize reflectivity effects and experimentally verified for a variety of configurations. Transmission electron microscopy (TEM) images for ZnO and Si QDs were obtained using a JEOL 2010-F microscope operating at $200 \mathrm{kV}$ (see figure 2). The IV performance of the solar cells, including open circuit and short-circuit conditions, was collected using an Orien Sol2A solar simulator under AM1.5G illumination and standard test conditions of $100 \mathrm{~mW} / \mathrm{cm} 2$ at room temperature, and the data was analyzed to extract the power conversion efficiency. In addition, external quantum efficiency (EQE) versus wavelength data collection was performed with a Newport External Quantum Efficiency Measurement System (QEPVSI-B). The incorporation of these films on the window side of the photovoltaic devices triggered improvements in their performance. Specifically, the collected results indicate an improvement produced by the influence of the coatings of Si QD for 5000 or $6000 \mathrm{rpm}$, as well as the coating of $\mathrm{ZnO}$ QD while varying QD concentration. After the deployment of the first coating of Si QDs, the solar cells exhibited an increase in PCE, which increased even more with the second coating of ZnO QDs. Ostensibly, it is feasible to vary both QD concentration and film thickness to optimize the ultimate PCE value using numerical methods to calculate the reflection and transmission coefficients of the stratified structures. 
The combined effect of the coatings led to an increase in energy conversion efficiency (PCE) from $14.42 \%$ to $15.41 \%$. The increase represents an improvement of $\sim 6.8 \%$ in the power conversion efficiency (PCE) of the photovoltaic devices employed.

\section{References:}

[1] Carlson, D.E. and C.R. Wronski, Applied Physics Letters 28(11) (1976), p. 671.

[2] Semonin, O.E. et al, Science 334 (6062) (2011), p. 1530.

[3] Trindade, T., P. O'Brien, and N.L. Pickett, Chemistry of Materials 13(11) (2001), p. 3843.

[4] Wang, J. et al, Journal of Materials Chemistry B 2(27) (2014) p. 4338.

[5] Rodriguez-Paez, J. et al, Journal of the European Ceramic Society 21(7) (2001), p. 925.
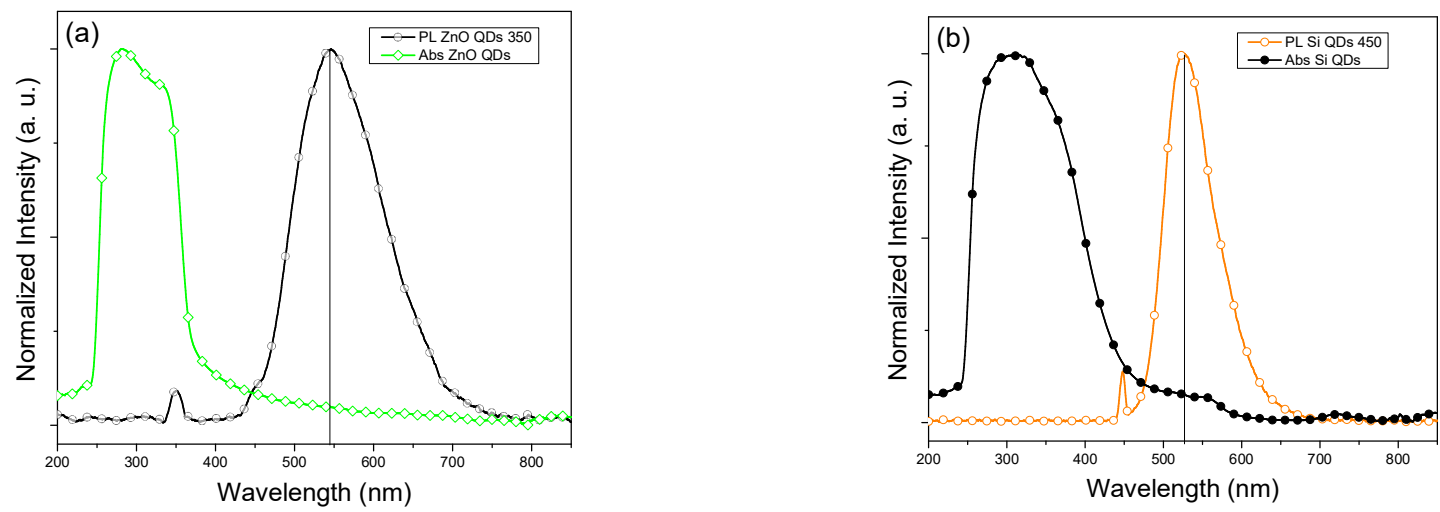

Figure 1. Absorption and photoluminescence spectra characterization with an excitation wavelength of $350 \mathrm{~nm}$ for ZnO QDs (a) and Si QDs (b).
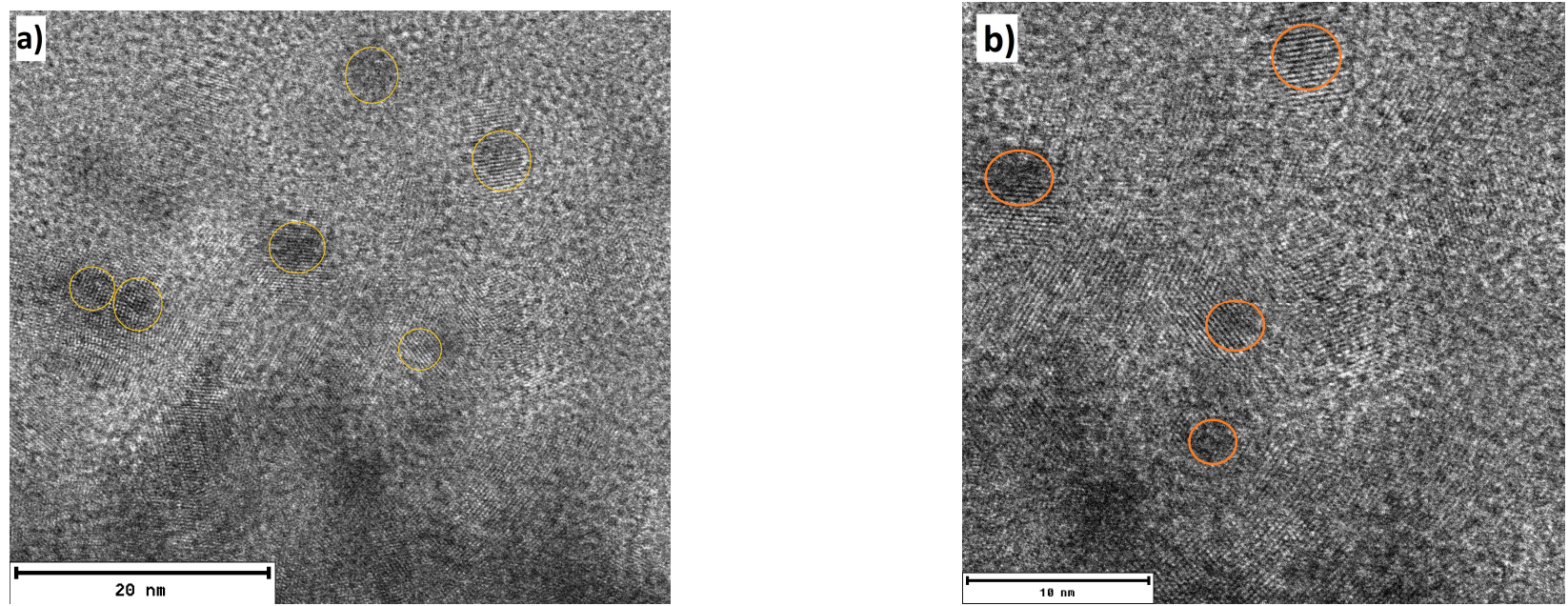

Figure 2. TEM images of $\mathrm{ZnO}$ QDs (a) and Si synthesized QDs (b). 